\title{
Environmental NanoBiology: Structural Evaluation Of Naturally Occurring Transition Metal Oxide-Containing Surface Films From Freshwaters
}

\author{
R. W. Smith and E. J. Sánchez \\ Department of Physics and the Environmental Sciences and Resources Program, Portland State \\ University, Portland, OR 97207-0751
}

Floating "iron-oxide" films are a common feature of wetlands, ephemeral pools, springs and seeps, some times called a mixed-valent iron-oxide film or schwimmeisen [1]. These silvery surface films are common in natural wetlands with iron rich sediments or mineral-rich waters. These films are also present at contaminated sites, rich in mixed iron oxides and containing other elements. The present paper describes structural investigations of these films and preliminary observations of some biological components of surface films as biological habitat. We demonstrate that other oxide minerals may be present, shedding some light on the presence of ferromanganese oxides in natural surface films. We also note that a complex structure is present in Environmental NanoBiology terms.

The collection of surface films followed several procedures for the collection of discrete samples (as opposed to bulk samples) on glass slides and cover slips and on methylcellulose pads. Similar methods were used to collect films on standard copper grids for Transmission Electron microscopy (TEM) analysis. The collection of surface films followed the procedures described earlier [2-4]. Most images were air-dried (except DIC images which were mounted in the wet state, not shown here), transported from the field on the day of the imaging. Scanning Electron Microscopy (SEM) was done on a JEOL 6300F following Au coating. TEM was done on an FEI Tecnai $\mathrm{G}^{2}$ F20 ST analytical microscope equipped with devices for Electron Energy Loss Spectroscopy (EELS) and Energy Dispersive X-ray Spectroscopy (EDXS). Polarizing light microscopy was done on a Leica system light microscope.

Typical optical and SEM images of the films are shown in Figs. 1 and 2, respectively. Bacterial activity was clearly present (Fig. 2). TEM images and a corresponding EDX spectrum are shown in Fig. 3 and 4, respectively. EDXS indicates that samples contain a mixture of iron and manganese oxides (Fig. 4) along with traces of organic material and other elements. The EELS data (not shown) confirm the mixed valences of Fe and $\mathrm{Mn}$ ions as suggested earlier [1], indicating incomplete oxidation states. The mixed-valent state of these films has been proposed [1][9].

In 2001, Grathoff et al. [1] proposed that the floating mixed valent iron films (proposed schwimmeisen) were a characteristic of ephemeral coastal pools, part of the Pleistocene dune sheet of coastal Oregon and Washington. Based on our current data, "schwimmeisen" may not be a proper descriptor for these films, which appear to be more complex than previously described. Our preliminary investigation demonstrates a much broader diversity of microscopic structure from a much wider diversity of habitats. Our research on such floating oxide films demonstrates variable biogeochemical processes at the air-water interface and in natural waters. The images presented here are a survey of microscopical methods to demonstrate different levels of complexity. We believe that a more intensive analysis is necessary to document the levels of complexity seen in these films. In environmental studies, contaminated environments often show a high level of iron mixed-valent films that may also absorb, adsorb and transport other elements such as manganese, as well as other minerals. Ferromanganese compounds are known from freshwater environments, but are relatively unstudied. The biogeochemical cycling of iron involves both transport and environmental reservoirs, Biogeochemical reservoirs of labile iron are important sources of nutrient iron [5][6][7][8]. 
References:

[1] Grathoff, G. H. et al. Clays and Clay Minerals 55 (2007) 635.

[2] Smith, R. W. and J. Dash. Microsc. Microanal. EMSA, Proc 49(Suppl. 2)(1989) 1004.

[3] Smith, R. W. In: SETAC, 12 Annual Meeting Seattle, Washington (Invited). 1991. Abs.

[4] Smith, R. W. Microsc. Microanal. 4 (Suppl. 2)(1999).

[5] Plachinda, P., R, W. Smith, S. Rouvimov et al. Microsc. Microanal. (2011) [This Volume]

[6] The generous support of P. Plachinda and S. Rouvimov is gratefully acknowledged for images and spectra, Figures 3 and 4.

[7] This research supported by the E. J. Sánchez NanoDevelopment Laboratory of the Department of Physics, Portland State University, P.O. Box 751-PHY, Portland, Oregon 97207.

[8] Portions of this research were supported by the Oregon Nanoscience and Microtechnologies Institute (ONAMI) grant to E. J. Sánchez. Portions of this research were supported by an instrument development grant, Office of Naval Research (ONR) to E. J. Sánchez.

[9] Smith, R. W. Proc. Coastal Zone 2007, Portland, Oregon CZ07, Ext. Abs., 3431.Smith.

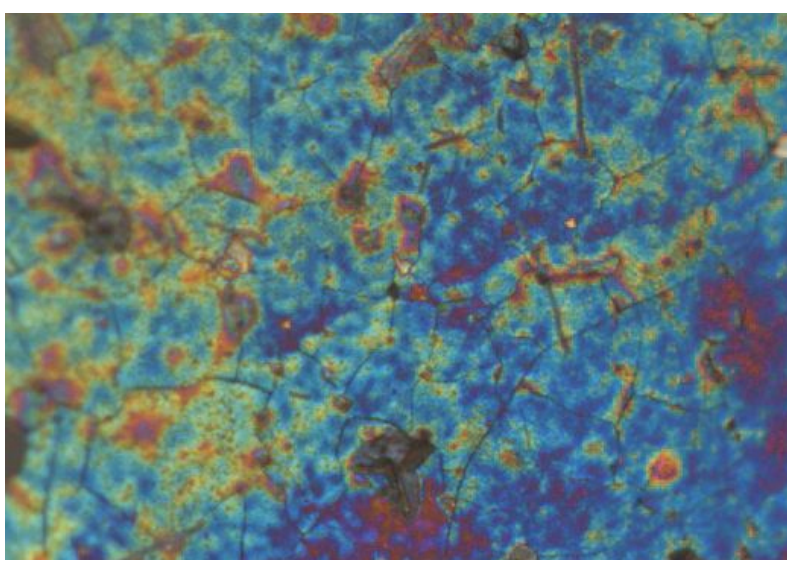

FIG. 1 Light microscopy shows the diverse character of mixed-valent iron films at low magnification.

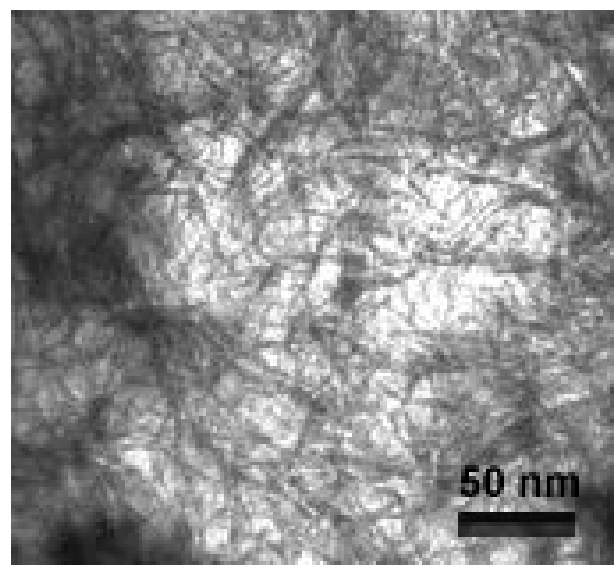

FIG. 3. Typical TEM bright field image of the iron-oxide film. Note the fibrous-like structure of the film.

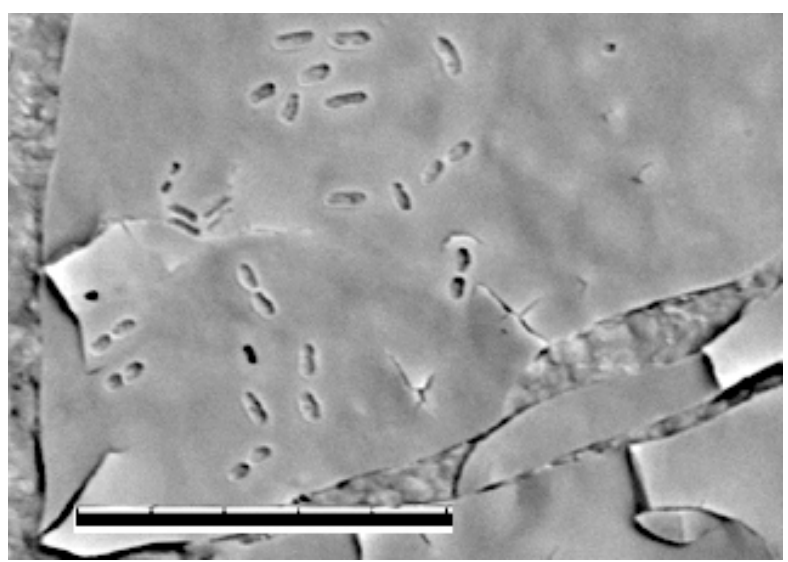

FIG.2 SEM image shows a platelet-like structure with evident bacterial pits. Bacteria were not fixed or preserved in this sample.

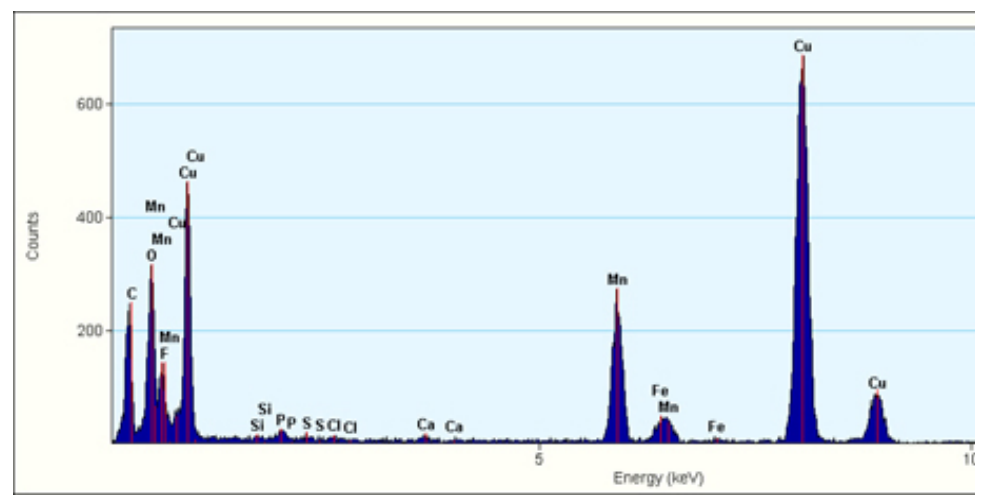

FIG 4. EDXS of the area in Fig. 1. Note: The presence of copper is due to TEM grid. Several dominant Fe peaks were also present in other samples, not shown. 